\title{
MÉTODO DA CONTINUAÇÃO APLICADO NA ANÁLISE DE CONTINGÊNCIA DE LINHAS DE TRANSMISSÃO
}

\author{
R. R. Matarucco*
}

J. H. Canossa*
D.A. Alves*

L.C.P. da Silva ${ }^{\dagger}$

\section{A. Castro ${ }^{\ddagger}$}

*UNESP - Departamento de Engenharia Elétrica, Av. Brasil, 56, Cx. P. 31, 15385-000 Ilha Solteira - Brasil

†UNICAMP - Faculdade de Engenharia Elétrica e de Computação, Cx. P. 6101, 13083-970, Campinas - Brasil

\section{RESUMO}

Este trabalho apresenta um método para a análise estática de contingências em Sistemas Elétricos de Potência utilizando o Método da Continuação. A margem de carregamento póscontingência é obtida a partir do ponto de máximo carregamento do caso base. A magnitude de tensão de uma barra qualquer é usada como parâmetro na etapa de parametrização do fluxo de carga continuado. O ramo selecionado para avaliação da contingência é parametrizado por um fator de escalonamento que possibilita a remoção gradual do ramo e assegura a convergência nos casos em que o método diverge para a retirada total da linha de transmissão. Em geral, para a maioria das contingências analisadas são necessárias poucas iterações para a determinação do ponto de máximo carregamento pós-contingência. Mostra-se que o método pode ser usado como uma técnica alternativa para a averiguação e até mesmo para a obtenção da lista de contingências críticas fornecida pela função de análise de segurança de sistemas elétricos.

\footnotetext{
Artigo submetido em $21 / 07 / 2004$

1a. Revisão em 22/02/2005

2a. Revisão em $26 / 10 / 2005$

3a. Revisão em 13/02/2006

4a. Revisão em 31/05/2006

Aceito sob recomendação do Editor Associado Prof. Glauco Nery Taranto
}

PALAVRAS-ChaVe: Método da Continuação, Colapso de Tensão, Fluxo de Carga, Ponto de Máximo Carregamento, Margem de Estabilidade de Tensão, Análise de Contingências.

\begin{abstract}
This work presents a new methodology for static contingency analysis of electric power systems by using Continuation Methods. The post-contingency loading margin is obtained from the base case maximum loading point. The voltage magnitude of any bus can be used as a parameter in the parameterization step of the proposed continuation power flow. The branch selected for contingency evaluation is parameterized by a scaling factor which allows its the gradual removal and assures the continuation power flow convergence for the cases where the method would diverge for the complete transmission line removal. In general, for most of the analyzed contingencies little iterations are necessary for the determination of the post-contingency maximum loading point. It is shown that the method can be used as an alternative technique to verify and even to obtain the list of critical contingencies supplied by the electric power systems security analysis function.
\end{abstract}

KEYWORDS: Continuation Methods, Voltage Collapse, 
Load Flow, Maximum Loading Point, Voltage Stability Margin, Contingency Analysis.

\section{INTRODUÇÃO}

Nos últimos anos uma grande quantidade de trabalhos têm sido dedicados ao desenvolvimento de métodos voltados para a análise de colapso de tensão. Os sistemas elétricos de potência (SEP) têm sido levados a operar bem próximos de seus limites de segurança, em conseqüência do aumento da demanda e do baixo investimento nos setores de geração e transmissão de energia elétrica. Tradicionalmente, os limites de segurança de operação eram associados aos problemas de limites térmicos dos equipamentos (transformadores e linhas de transmissão (LT)) e de estabilidade transitória. A limitação no transporte de energia estava, via de regra, intimamente relacionada à necessidade de transportar grandes blocos de energia através de grandes distâncias até os remotos centros de carga. Assim, surgia a restrição relacionada com a instabilidade angular transitória ou instabilidade de primeiro "swing". O emprego de relés e disjuntores de um ciclo para rápida eliminação de faltas, de sistemas de excitação de alto desempenho e outros controles, aumentaram os limites de transferência desses sistemas. Também, esses limites tornaram-se menos restritivos à medida que os sistemas tornaram-se mais malhados. Posteriormente, a estabilidade de tensão tornou-se um fator limitante para muitos sistemas em virtude da falta de suporte de potência reativa necessário para se garantir um perfil de tensão satisfatório para o sistema, o que tem resultado na limitação da utilização plena dos recursos de transmissão, particularmente nas interligações, e muitas vezes a perda de uma região ou blecaute do sistema (Taylor, 1994).

As ações de controle efetuadas durante a operação em temporeal de um SEP não devem somente objetivar um melhor aproveitamento dos recursos de geração e de capacidade de transmissão, mas também devem evitar que o sistema seja levado a operar próximo a uma condição crítica onde, por exemplo, um pequeno aumento de carga ou uma contingência possa causar o colapso de tensão. Assim, nas etapas de planejamento e operação de um SEP, devem ser definidas as margens de estabilidade de tensão e as ações de controle necessárias não só para as condições normais de operação (caso base), mas também para diferentes condições de contingência.

A margem para o colapso de tensão é definida como o maior aumento de carga que um sistema pode suportar a partir de um dado ponto de operação (IEEE-SSC, 1999), conforme mostra a figura 1. Em termos de aumento de carga, a margem definida como a diferença entre o ponto de operação de précontingência (ponto $\mathrm{O}$ ) e o ponto de máximo carregamento de pós-contigência $\left(\mathrm{PMC}_{\text {pós }}\right)$ é utilizado como índice para a

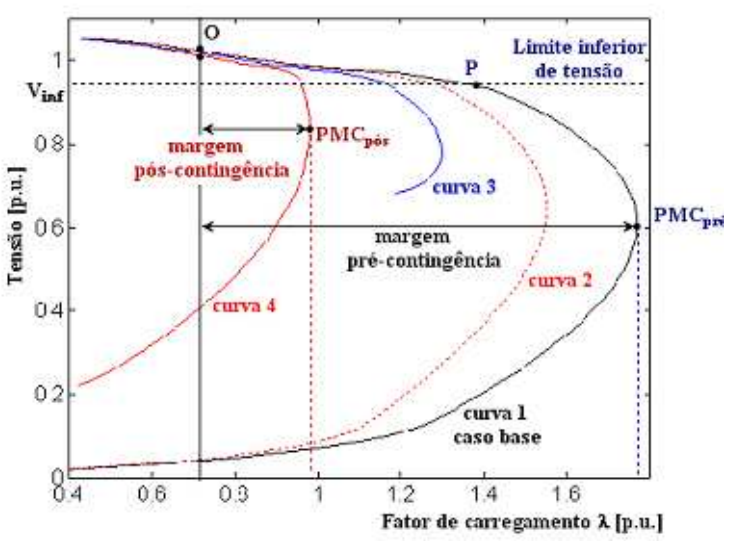

Figura 1: Definição da margem de carregamento de pré e pós-contingência.

análise de estabilidade de tensão. O Western System Coordinating Council (WSCC) requer que seus membros garantam pelo menos $5 \%$ de margem de potência ativa em qualquer situação de contingência simples (WSCC, 1998). Essa política também tem sido recomendada pelas empresas do setor elétrico nacional (FTCT-Força Tarefa "Colapso de Tensão", 1999).

Antes da análise de estabilidade de tensão realiza-se a seleção e a ordenação das contingências de acordo com o impacto que estas causam ao sistema. O objetivo é reduzir o tempo computacional.

A avaliação da estabilidade de tensão através do traçado de curvas $\mathrm{P}-\mathrm{V}$ e $\mathrm{Q}-\mathrm{V}$ para cada contingência e várias condições operativas é baseada em métodos de análise estática (Taylor, 1994). O levantamento de ambas as curvas, P-V e Q-V, é a metodologia recomendada pelo WSCC para assegurar que a margem mínima requerida seja atendida. Já o Operador Nacional do Sistema Elétrico Brasileiro (ONS), considera o traçado da curva $\mathrm{P}-\mathrm{V}$ como a metodologia mais apropriada para a determinação da margem de estabilidade, e o levantamento da curva Q-V como uma metodologia complementar para avaliar as margens de potência reativa e os locais para o reforço do sistema (ONS, 2001). Como resultados deste estudo definem-se as ações preventivas e corretivas necessárias para se garantir a estabilidade.

O método de Fluxo de Carga (FC) utilizado nestas análises deve ser eficiente e confiável não só a fim de ser considerado atrativo para os estudos de planejamento, mas também para atender os requisitos necessários para as aplicações de operação em tempo-real. As curvas $\mathrm{P}-\mathrm{V}$ e $\mathrm{Q}-\mathrm{V}$ são traçadas calculando-se as soluções do FC para sucessivos incrementos de carga e geração, ao longo de uma direção predefinida, até que o PMC seja encontrado. Tradicionalmente, 
assume-se que o aumento de carga de uma determinada área é feito com fator de potência constante e proporcional ao carregamento do caso base com modelo de carga de potência constante. As principais razões para se adotar essa modelagem para a carga são (IEEE-PSSC, 1999; Cañizares, 1995; Flatab $\emptyset$, 1990; Chiang et alii, 1999):

1. os estudos têm mostrado que os modelos algébricos (estáticos) do sistema de potência podem ser suficientes para as análises de colapso de tensão de longo prazo;

2. as ações de diversos dispositivos de um sistema de potência (p.ex., os transformadores com comutação de taps sob carga - OLTC) procuram restaurar as tensões aos seus respectivos valores de pré-distúrbio e assim, fazem com que as cargas dependentes das tensões atuem como independentes das tensões nas condições de regime permanente (estáticas); e

3. o fato de que esse modelo, quando comparado ao modelo de carga dependente da tensão, mostra-se mais conservador, posto que resulta em margens de carregamento (MC) menores que as reais.

Dessa forma, no que se refere às análises de colapso de tensão, essa modelagem fornece condições operacionais mais seguras para o sistema. Freqüentemente, nestas análises, o FC permite a obtenção de uma solução, mas em alguns casos isto não acontece, mesmo quando do uso de cálculos com dupla-precisão e de algoritmos anti-divergentes. A divergência do FC é freqüentemente adotada como um critério de instabilidade, entretanto, esta também pode ser conseqüência de problemas puramente numéricos uma vez que a matriz Jacobiana das equações do FC torna-se singular no PMC. Assim o uso do FC fica restrito à parte superior da curva P-V (correspondente à operação estável). A divergência do FC também pode ser devida a ocorrência de contingências para as quais o PMC de pós-contingência ( $\mathrm{PMC}_{\text {pós }}$ ) na curva 4 da figura 1 é menor que o carregamento do ponto de operação do caso base (ponto $\mathrm{P}$, por exemplo). Portanto, nesses casos, o algoritmo de FC deverá averiguar se existe um ponto de operação factível para tais condições de carregamento e contingência em particular, e realizar as ações corretivas para que seja restabelecida a margem necessária. Em sistemas de maior porte fica difícil aferir sobre a existência ou não de um ponto de operação devido às várias causas da divergência de um FC, tais como: a existência de múltiplas soluções, a deficiência do método utilizado na resolução, a existência de singularidades, a estimativa inicial, a inexistência da solução, etc.

Os métodos de Fluxo de Carga Continuado (FCC) superam as dificuldades numéricas acima mencionadas pela adição de equações parametrizadas. A reformulação das equações de FC visaCoeficiente de Progressão (CP): 0.9419 Coeficiente de Progressão Futuro (CPF): remover a singularidade da matriz no PMC e, por conseguinte, eliminar os problemas numéricos que surgem em sua vizinhança. Dessa forma, possibilitam que algoritmos com precisão simples sejam usados tanto na vizinhança quanto no próprio PMC. Essa é a razão principal de sua ampla utilização para o traçado das curvas P-V (Ajjarapu e Christy, 1992; Chiang et alii, 1995). Assim sendo, o cálculo das margens de estabilidade usando o FC, ou FCC, são procedimentos que consomem um tempo computacional relativamente alto quando um grande número de contingências deve ser analisado. Em função disso, muitos trabalhos relevantes têm procurado um algoritmo rápido e preciso de seleção e classificação de contingências de acordo com o grau de severidade, o qual forneça uma lista pequena de contingências críticas a partir de uma lista grande de contingências possíveis.

Em Ejebe et alii (1996) e Chiang et alii (1997) foram propostos métodos para estimar ou calcular rapidamente a margem através do uso de técnicas de ajuste de curvas que requerem três soluções do FC por contingência. A principal desvantagem destes método é ser fortemente dependente da forma da curva $\mathrm{P}-\mathrm{V}$ podendo falhar nos casos em que são considerados os limites de tap transformadores (OLTC) e de potência reativa nas barras de geração. Além disso, é necessária uma solução próxima ao PMC e a identificação de uma barra cuja curva $\mathrm{P}-\mathrm{V}$ apresente uma geometria adequada para o ajuste de curva. Em Yokoyama e Sekine (1989) a aproximação de primeira ordem para a margem do sistema é calculada usando os gradientes de tensão determinados em um par de soluções do FC (uma estável, solução de alta tensão, e uma instável, solução de baixa tensão) próximo do PMC. Contudo, os resultados apresentados em Ejebe et alii (1996) mostram que a precisão desse método não é satisfatória. Em Van Cutsem e Mailhot (1997) foi proposto uma aproximação de segunda ordem para a curva Q-V. O método apresentado em Greene et alii (1999) usa sensibilidades linear e quadrática da margem P-V para obter cálculos rápidos para a margem de póscontingência. Embora seja um método muito rápido e portanto razoável para a aplicação na seleção de contingências, os resultados mostraram que as margens obtidas eram praticamente inaceitáveis para muitas das contingências estudadas.

Um outro aspecto importante relacionado com o FCC é que a sua convergência está associada ao parâmetro escolhido e à trajetória de solução. Dependendo do parâmetro da continuação escolhido o FCC pode não convergir, p. ex., para certos carregamentos no caso base (ponto $\mathrm{P}$ da figura 1) e contingências (curvas 3 e 4 da figura 1) o PMC pós-contingência é menor que o carregamento do ponto de operação do caso base, e nessa condição, uma vez que não existe um ponto de operação pós-contingência, o uso do fator de carregamento $(\lambda)$ como parâmetro acarretará na divergência do FC bem 
como do FCC. Na técnica para a determinação da margem proposta em Feng e Xu (2000), determina-se primeiramente

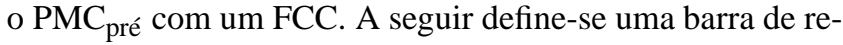
ferência cuja magnitude de tensão de pós-contingência será fixada (adotada como parâmetro), enquanto que $\lambda$ será considerado como variável no FCC. A partir dessas considerações, aplica-se a contingência e calcula-se o $\mathrm{PMC}_{\text {pós }}$ e a respectiva margem. Nos casos em que esse procedimento falhar, como seria o caso da curva 3 da figura 1, o método de Newton modificado é usado para encontrar uma barra de referência e sua respectiva magnitude de tensão para um ponto próximo do $\mathrm{PMC}_{\text {pós. }}$. Dessa forma, uma vez que a barra crítica de pós-contingência, bem como sua magnitude de tensão, não são conhecidas a priori, e a sua identificação é importante para a obtenção de uma solução, a determinação da margem de estabilidade pode se tornar um processo problemático e computacionalmente oneroso quando a magnitude da tensão é escolhida como parâmetro nas análises de contingências.

Neste trabalho são apresentadas as dificuldades numéricas que podem surgir quando do uso dos métodos de FC e FCC para a determinação da MC pós-contingência em SEP. O sistema IEEE de 14 barras (Freris e Sasson, 1968) é utilizado para exemplificar os possíveis problemas que podem surgir durante a análise de contingência de LT. Para esse sistema é apresentado um caso de contingência que acarreta a divergência destes métodos. O FCC que utiliza a magnitude de tensão de barra como parâmetro é usado para a avaliação dos efeitos da remoção da LT. A seguir é apresentado o método proposto para evitar a divergência do método do FCC durante a análise de qualquer contingência envolvendo uma LT.

Observa-se que o método torna fácil o desenvolvimento e a implementação do método da continuação para a análise estática de contingências em sistemas elétricos de potência, isto é, as contingências são simuladas com eficiência e sem grandes modificações dos algoritmos de FCC. Assim, o método pode ser usado como uma técnica alternativa não só para a averiguação, mas até mesmo para a obtenção da lista de contingências críticas fornecida pela função de análise de segurança de sistemas elétricos. O método foi aplicada aos sistemas do IEEE de 14, 57 (Freris e Sasson, 1968) e 118 barras (Web site da Universidade de Washington).

\section{FLUXO DE CARGA CONTINUADO}

A idéia básica do fluxo de carga continuado (FCC) consiste na obtenção de sucessivas soluções de FC objetivando traçar os perfis de tensão a partir de um caso base, até o PMC. Obtém-se, com isso, não só a margem de carregamento, mas também, informações adicionais a respeito do comportamento das tensões das barras do sistema em análise. Em geral as equações de FC podem ser escritas como

$$
\mathbf{G}(\mathbf{V}, \theta, \lambda)=\mathbf{0}
$$

onde $\mathbf{V}$ é o vetor das magnitudes de tensões nodais, $\theta$ é o vetor dos ângulos de fase nodais, $\lambda$ é o fator de carregamento e $\mathbf{G}$ é um vetor composto pelas equações dos balanços de potências ativa e reativa nodais. A equação (1) pode ser reescrita como

$$
\begin{aligned}
& \lambda \mathbf{P}^{e s p}-\mathbf{P}(\theta, \mathbf{V})=\mathbf{0} \\
& \lambda \mathbf{Q}^{e s p}-\mathbf{Q}(\theta, \mathbf{V})=\mathbf{0}
\end{aligned}
$$

onde $\mathbf{P}^{e s p}=$ Pgen - Pcarga é a diferença entre as potências ativas gerada e consumida para as barras de carga (PQ) e de geração (PV), $\mathbf{Q}^{e s p}=-\mathbf{Q c a r g a}$ para as barras $\mathrm{PQ}$, e $\lambda=1$ corresponde ao carregamento do caso base. Observa-se que no caso das barras de geração, quando o limite de potência reativa (Qlim) é atingido a barra passa a ser tratada da mesma forma que a barra de carga, sendo que Qgen=Qlim. Nesse caso, $\lambda$ deverá multiplicar apenas o Qcarga, isto é, $\mathbf{Q}^{e s p}=$ Qgen - $\lambda$ Qcarga.

Para uma certa barra $k, \mathbf{P}(\theta, \mathbf{V})$ e $\mathbf{Q}(\theta, \mathbf{V})$ são dados por:

$$
\begin{aligned}
& P_{k}(\theta, V)=G_{k} V_{k}^{2}-V_{k} \sum_{l \in \Omega_{k}} V_{l}\left(g_{k l} \cos \theta_{k l}+\right. \\
& \left.b_{k l} \operatorname{sen} \theta_{k l}\right), k \in P Q, P V \\
& Q_{k}(\theta, V)=-B_{k} V_{k}^{2}-V_{k} \sum_{l \in \Omega_{k}} V_{l}\left(g_{k l} \operatorname{sen} \theta_{k l}-\right. \\
& \left.b_{k l} \cos \theta_{k l}\right), k \in P Q .
\end{aligned}
$$

onde $\Omega_{k}$ é o conjunto de todas as barras diretamente conectadas à barra $k$. $\left(G_{k}+j B_{k}\right)$ é o elemento da diagonal $(k, k)$ da matriz admitância nodal e $\left(g_{k l}+j b_{k l}\right)$ corresponde à admitância série do ramo que conecta as barras $k$ e $l$. O sistema de equações (2) pressupõe que o carregamento da rede é proporcional ao do caso base e considera o fator de potência constante. $\mathbf{P}^{e s p}$ e $\mathbf{Q}^{e s p}$ podem ser, respectivamente, definidos como sendo iguais a $\left(\mathbf{K}_{\mathbf{p}} \mathbf{S} \cos \varphi\right)^{\text {esp }}$ e $\left(\mathbf{K}_{\mathbf{q}} \mathbf{S} \sin \varphi\right)^{\mathbf{e s p}}$ (Ajjarapu e Christy, 1992). Assim, é possível realizar uma variação de carregamento individual, isto é, para cada barra do sistema, considerando para cada uma, um crescimento de carga com fatores de potência diferentes aos do caso base. Tradicionalmente, entretanto, assume-se que o aumento de carga de uma determinada área é feito com fator de potência constante e proporcional ao carregamento do caso base com modelo de carga de potência constante, visto que este fornece a condição operacional mais segura para o sistema (WSCC, 1998).

Uma vez definido um padrão de variação da carga e uma estratégia de despacho da geração, é necessário saber o quanto 
a demanda poderá aumentar antes que o sistema entre em colapso, ou seja, qual é a MC para as condições preestabelecidas. Para isso realiza-se o traçado da curva P-V por meio de sucessivas soluções do sistema (2) utilizando o método de Newton e considerando um crescimento da carga na direção predefinida. Nesse procedimento, as potências ativas $(\mathbf{P})$ e reativas $(\mathbf{Q})$ nas barras de carga são as variáveis independentes, enquanto que as magnitudes de tensão $(\mathbf{V})$ e os ângulos de fase $(\theta)$ são as variáveis dependentes.

Com a inclusão de $\lambda$ como variável na equação (2) o sistema resultará em $n$ equações e $n+1$ incógnitas. Assim, qualquer uma das $n+1$ incógnitas pode ser definida como parâmetro. Quando $\lambda$ é usado como parâmetro o sistema (2) passa a ter novamente $n$ equações e $n$ incógnitas. O seu valor é incrementado gradualmente (ver figura 2$)$ a partir do caso base $(\lambda$ $=1$ ), até um valor para o qual não mais se obtenha solução (o processo iterativo do FC não converge). Nesse ponto realizase um controle de passo que consiste numa simples redução no incremento de $\lambda \mathrm{e}$ a solução de um novo $\mathrm{FC}$ a partir da última solução convergida. O PMC é considerado como sendo o último ponto convergido, após sucessivas repetições desse procedimento. Entretanto, conforme já comentado, a divergência do FC é conseqüência da singularidade da matriz Jacobiana (J) de (2) no PMC e, portanto, não será possível determiná-lo precisamente. Em Alves et alii (2004) mostrouse que diferentes valores de PMC eram obtidos quando do uso dos FC convencionais de Newton e desacoplado rápido. Além da incerteza a respeito do ponto obtido ser realmente o PMC, as sucessivas reduções no passo (incremento do parâmetro $\lambda$ ) podem resultar num processo computacional lento e oneroso quando comparados aos métodos de FCC. Diversos autores propuseram diferentes implementações dos conhecidos métodos da continuação para superar as dificuldades numéricas introduzidas pela singularidade da matriz $\mathbf{J}$ e possibilitar a determinação do PMC (Ajjarapu e Christy, 1992; Chiang et alii, 1995; Alves et alii, 2002). Entre os muitos métodos descritos na literatura, o mais amplamente utilizado consiste de quatro elementos básicos: um procedimento de parametrização, um passo preditor, um controle de passo e um passo corretor.

A partir da solução da equação (2) para o caso base $\left(\mathbf{V}^{0}, \theta^{0}\right.$, $\lambda^{0}=1$ ), um passo preditor é executado para encontrar uma estimativa para a próxima solução. Os preditores mais utilizados são o tangente e o secante. Conforme pode-se ver na figura 3 em que $\lambda$ é usado como parâmetro, no preditor tangente a estimativa é encontrada através de um passo de tamanho apropriado na direção do vetor tangente à curva $\mathrm{P}-\mathrm{V}$ no ponto correspondente à solução atual (Ajjarapu e Christy, 1992). Da mesma forma que no caso do FC considerando incrementos fixos no valor de $\lambda$, o FCC também irá apresentar problemas numéricos nas proximidades do PMC.

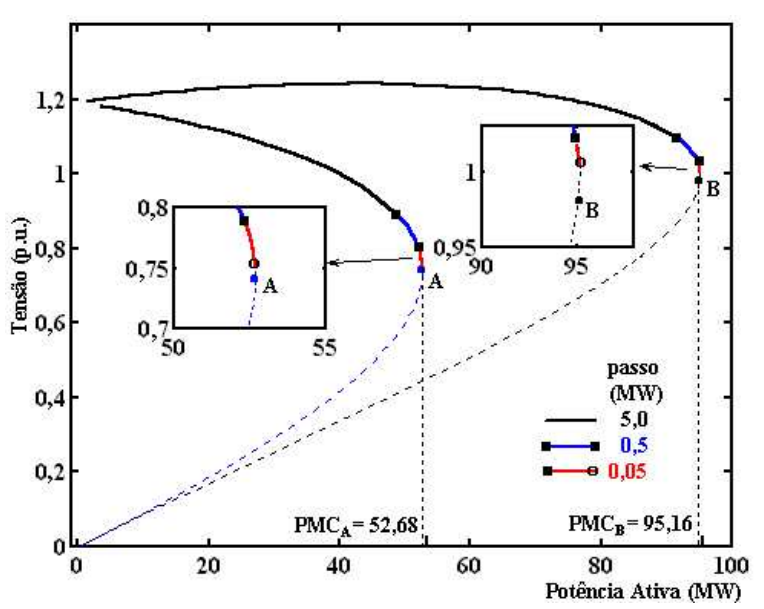

Figura 2: Uso do FC para o traçado das curvas $\mathrm{P}-\mathrm{V}$ para duas condições de variação da carga e de despacho da geração, considerando sucessivos incrementos para $\lambda$.

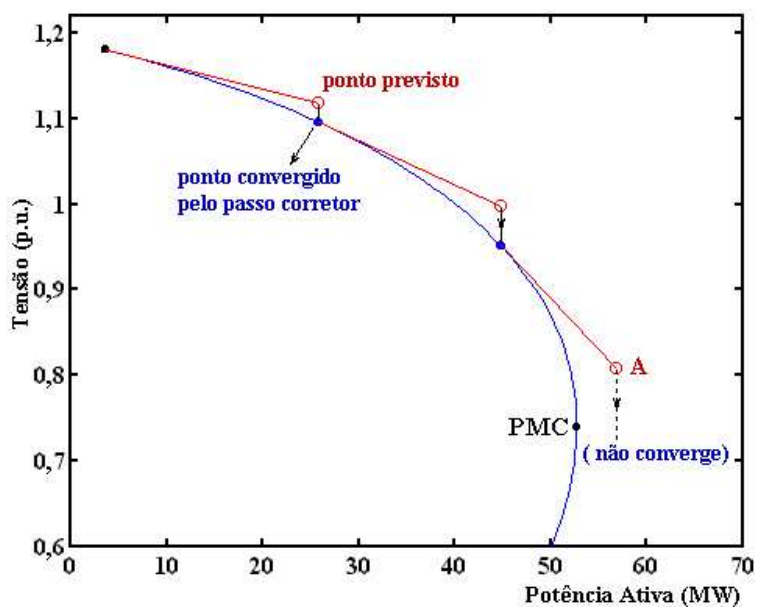

Figura 3: FCC com preditor tangente usando $\lambda$ como parâmetro da continuação.

São utilizados dois métodos secantes: o de primeira ordem, que usa as soluções atual e anterior, para estimar a próxima, e o de ordem zero, que usa a solução atual e um incremento fixo no parâmetro $\left(\lambda, \theta_{k}\right.$, ou $\left.V_{k}\right)$ como uma estimativa para a próxima solução (Chiang et alii, 1995). A parametrização fornece uma forma de identificar cada solução ao longo da trajetória a ser obtida. A parametrização local consiste na troca de parâmetro próximo ao PMC (Ajjarapu e Christy, 1992). A variável escolhida é aquela que apresentar a maior variação no preditor tangente, e no secante, a que apresentar a máxima variação relativa. A partir daí $\lambda$ passa a ser tratado como variável dependente, enquanto que a variável escolhida passa a ser o novo parâmetro. O sistema (2) permanece com $n$ equações e $n$ incógnitas. 
Finalmente, após se efetuar a previsão, torna-se necessário realizar a correção da solução aproximada para obter-se a solução final. Na maioria das vezes o ponto obtido pelo passo preditor está próximo da solução correta e assim, poucas iterações são necessárias no passo corretor para a obtenção da solução correta, dentro da precisão desejada. O método de Newton é o mais usado no passo corretor, embora outro método numérico como o apresentado em Alves et alii (2004) também possa ser utilizado. Neste passo uma equação do tipo $y-y^{e s t}=0$ onde $y$ e $y^{\text {est }}$ correspondem à variável escolhida como parâmetro de continuação e seu respectivo valor estimado, obtido pelo passo preditor, pode ser acrescentada ao sistema (1), ou o valor do parâmetro, como é feito no caso do preditor de ordem zero, pode ser simplesmente fixado em $y^{e s t}$.

\section{CARACTERIZAÇÃO DO PROBLEMA}

A utilização do método da continuação para o traçado da correspondente curva $\mathrm{P}-\mathrm{V}$ de cada contingência é um processo que consome muito tempo computacional. Também, a convergência do FCC dependerá da contingência e do parâmetro adotado na etapa de parametrização. A figura 4 apresenta quatro curvas P-V (tensão da barra $14, V_{14}$, em função do fator de carregamento $\lambda$ ) do sistema IEEE 14 barras: curva 1 , correspondente ao caso base; curva 2 , para a contingência de uma das LT entre as barras 1 e 2; curva 3, para a contingência da LT entre as barras 2 e 3; curva 4, para a contingência das duas LT entre as barras 1 e 2 . Nesta figura pode se observar melhor algumas das prováveis dificuldades que podem surgir quando do uso dos FC e FCC para análise de contingências. As curvas foram obtidas usando um FCC apresentado em Alves et alii (2002). Considere por exemplo o ponto P na curva de pré-contingência, como sendo o ponto de operação do caso base. De acordo com a definição da MC, o sistema apresenta neste caso uma MC positiva (MC>0). Três contingências serão analisadas, uma em que o sistema permanecerá operando com uma MC positiva (curva 2), e outras duas em que a MC será negativa (curvas 3 e 4). Se o carregamento $(\lambda)$ do sistema for mantido fixo, isto é, $\lambda$ for adotado como parâmetro, no primeiro caso (curva 2) o sistema permanecerá estável e passará a operar no ponto A, enquanto que nos demais (curvas 3 e 4), entrará em colapso porque não existirá solução factível para este carregamento; o FCC divergirá. O mesmo ocorrerá quando do uso do FC.

Portanto, nos casos em que a MC for negativa, será necessário estabelecer uma política para corte de carga a fim de que o sistema possa continuar em operação. Utilizando o FCC parametrizado por $\lambda$, a determinação da MC só será possível para a curva 2. A determinação das demais MC não será possível. Por outro lado, no caso da utilização da tensão do ponto P como parâmetro da continuação no FCC, os novos pontos

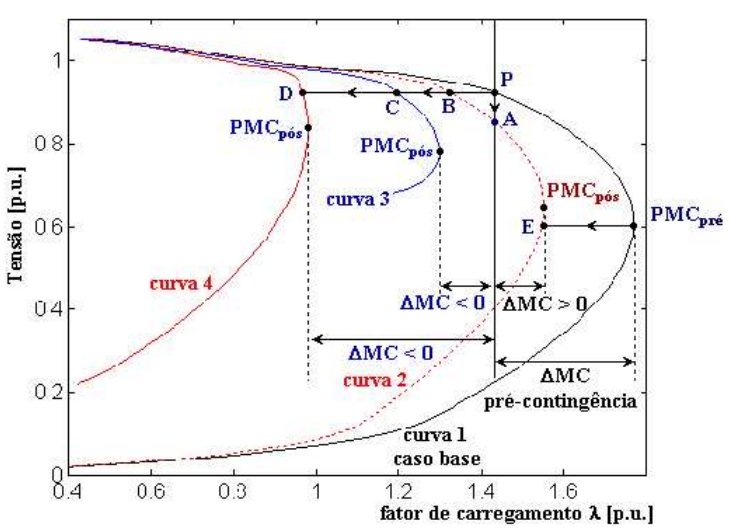

Figura 4: Curvas P-V para contingências de LT do sistema IEEE-14 barras: curva 1, caso base; curva 2, contingência de uma das duas LT entre as barras 1 e 2 ; curva 3, contingência da LT entre as barras 2 e 3; curva 4, contingência das duas LT entre as barras 1 e 2 .

de operação de pós-contingência seriam os pontos $\mathrm{B}, \mathrm{C}$, e $\mathrm{D}$, respectivamente. Assim, as correspondentes MC poderiam ser determinadas usando o FCC parametrizado por $\lambda$ ou pela tensão para a obtenção dos respectivos $\mathrm{PMC}_{\text {pós }}$. Entretanto, dado o grande número de contingências a serem analisadas num sistema real, bem como o fato de que a grande maioria dessas contingências resultam em curvas P-V similares à curva 2, i.e., cujos $\mathrm{PMC}_{\text {pós }}$ são muito próximos ao $\mathrm{PMC}_{\text {pré }}$, esse procedimento resultaria num processo computacional muito lento se for utilizado para a seleção e classificação das contingências. Com base na argumentação de que

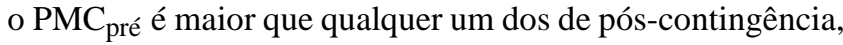
e que estes, por sua vez, são muito próximos àquele, (Feng e Xu, 2000) propuseram uma técnica para a determinação da $\mathrm{MC}$ pós-contingência a partir do $\mathrm{PMC}_{\text {pré }}$. Uma vez definido o padrão de crescimento de carga e a estratégia de des-

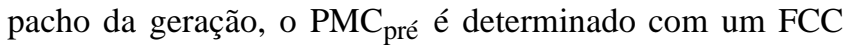
(Ajjarapu e Christy, 1992; Alves et alii, 2002). A seguir, define-se uma barra de referência cuja magnitude de tensão pós-contingência será fixada (adotada como parâmetro), enquanto que $\lambda$ será considerado como variável no FCC. A partir dessas considerações, aplica-se a contingência e calculase o $\mathrm{PMC}_{\text {pós }}$ e a respectiva margem. Observa-se que para muitas contingências a tensão crítica de pré-contingência é praticamente igual à de pós-contingência e assim, o ponto $\mathrm{E}$ (curva 2, figura 4) seria o próprio $\mathrm{PMC}_{\text {pós }}$. Quando isso não ocorre, i.e., quando o seu valor de pré-contingência é uma aproximação do valor da de pós-contingência, o valor de $\lambda$ será próximo ao do correspondente $\mathrm{PMC}_{\text {pós }}$. Nesse caso pode ser necessário um refinamento para se obter seu valor exato, o que é feito partindo do ponto E, e utilizando o FCC usando $\lambda$ ou a tensão como parâmetro. O problema 
do cálculo da MC transforma-se num problema de escolha da barra de referência e da estimativa do valor de sua tensão de pós-contingência. Conforme se pode ver na figura 5(b), na aplicação desse método para determinação do $\mathrm{PMC}_{\text {pós }}$ da curva 2 foram necessárias 9 iterações, sendo 3 iterações para a obtenção do ponto $\mathrm{E}$, e, na fase de refinamento, mais 6 iterações para se obter o $\mathrm{PMC}_{\text {pós }}$ usando a tensão da barra 14 como barra de referência (parâmetro) e um passo de +0.01 p.u.. Para essa contingência a redução na MC foi de $28,35 \%$. $\mathrm{Na}$ figura 5(c) pode se ver que o número de iterações necessárias para se obter o $\mathrm{PMC}_{\text {pós }}$, partindo do caso base $(\lambda=1)$ convergido e aplicando contingência da LT, foi igual a 71 . Portanto, para a maioria das contingências o número total de iterações necessárias para se determinar o PMC a partir

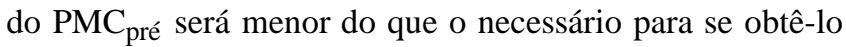
partindo do caso base $(\lambda=1)$ convergido e aplicando contingência da LT. Observa-se ainda que para alguns casos, a obtenção do $\mathrm{PMC}_{\text {pós }}$ considerando a aplicação da contingência no caso base convergido pode falhar devido à inexistência de solução.

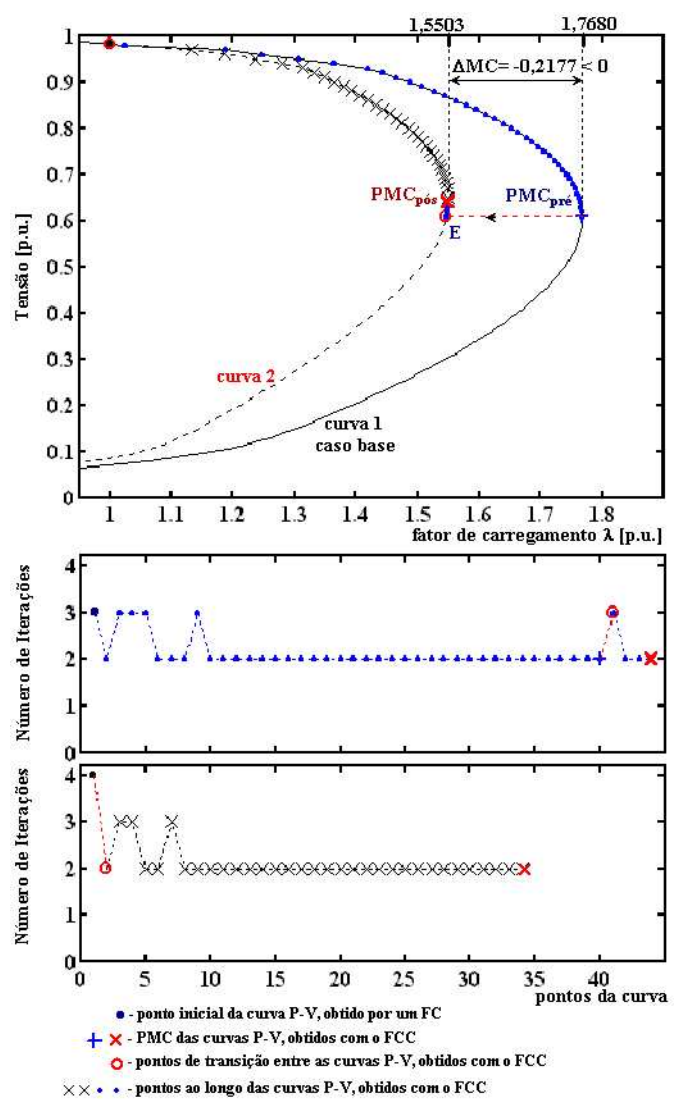

Figura 5: (a) Curvas P-V para o caso base e para a contingência de uma das duas LT entre as barras 1 e 2 do sistema IEEE-14; (b) número de iterações por ponto da curva; e (c) número de iterações, partindo do caso base $(\lambda=1)$ convergido e aplicando contingência da LT.
No caso da curva 4, conforme se pode ver na figura 6 , foram necessárias ao todo 50 iterações, 4 iterações para obter o ponto E, e 46 iterações na fase de refinamento. A redução na $\mathrm{MC}$ foi de $102,47 \%$, i.e., $\mathrm{MC}<0$. Nos casos em que esse procedimento falhar, como é o caso da curva 3 da figura 4, propõe-se o uso de um método de Newton-Raphson com fator de amortecimento, que consiste no uso de um fator de passo para atualizar as variáveis do $\mathrm{FC}$ de forma a prevenir a divergência do processo iterativo e atingir a superfície entre as regiões de existência e de não existência da solução das equações do FC (Feng e Xu, 2000; Castro e Braz, 1997; Overbye, 1994). O valor ótimo do passo é calculado de forma a minimizar uma função quadrática. Para os casos em que não há solução real para as equações do FC, o método evita a divergência do FC com o fator de passo tendendo a zero. Em Feng e Xu (2000) o método de NewtonRaphson com fator de amortecimento é usado para encontrar uma barra de referência e estimar o valor de sua magnitude de tensão de pós-contingência. O valor ótimo do passo também é calculado de forma a minimizar uma função quadrática, porém é proposto um novo esquema para a seleção do valor ótimo do fator de amortecimento de passo.

Para os casos em que não há convergência quando da contingência, isto é, quando a carga não pode ser atendida após a contingência, um algoritmo de Fluxo de Potência Ótimo

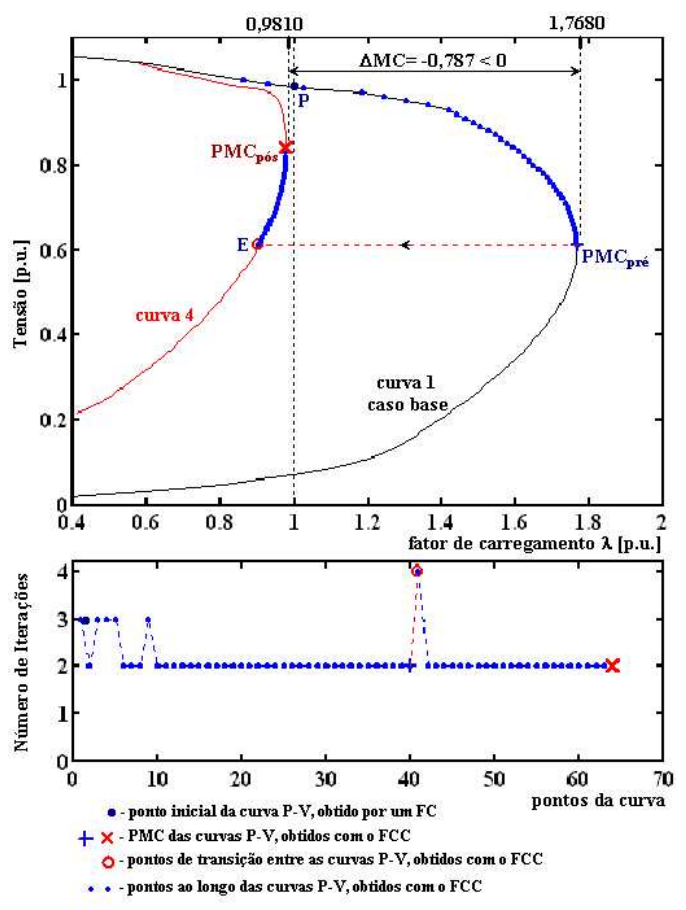

Figura 6: (a) Curvas P-V para o caso base e para a contingência das duas LT entre as barras 1 e 2 do sistema IEEE-14; e (b) número de iterações por ponto da curva. 
(FPO) é utilizado para a obtenção do mínimo corte de carga que atenda as restrições de igualdade (equações do fluxo de carga), bem como restrições de desigualdades (tais como os limites de tensão e de tap de transformadores, restrições operacionais). Diversas técnicas são encontradas na literatura recente, entre as quais podem-se citar Granville et alii (1996), Torres et alii (2002), Barbosa e Salgado (2001) e Rider et alii (2004). Em geral as restrições de desigualdades não são tratadas da mesma forma no FC ou no FCC. Assim, por exemplo, nesse estudo, conforme é comentado em Granville et alii (1996-2), a fim de comparação com o FCC, o FPO deveria entre outras, seguir a mesma lógica existente nos programas FC e FCC com relação a mudança do tipo de barra PV-PQ quando a geração de potência reativa atinge seu limite. Os resultados obtidos pela aplicação do método da continuação proposto foram comparados aos obtidos através de um FPO. O objetivo dessa comparação foi observar a precisão dos resultados obtidos por diferentes abordagens. O FPO foi adaptado a partir do apresentado em da Costa et alii (1998), o qual é baseado no método de Newton modificado associado à função Lagrangeana aumentada. Neste método a matriz Lagrangeana é rearranjada de forma a manter-se a estrutura de matriz incidência por partes (a mesma da matriz admitância) e possibilitar que as operações matemáticas sejam feitas entre elementos e não mais entre submatrizes quadradas de ordem 4. Tal procedimento, além de permitir explorar-se de forma mais eficiente a esparsidade da matriz Lagrangeana, reduz o número de operações aritméticas em aproximadamente $60 \%$ daquele correspondente ao do uso de submatrizes. Outra modificação introduzida pelo método na matriz Lagrangeana foi a de se considerar apenas os elementos da diagonal da matriz Hessiana, enquanto que as informações de primeira ordem, correspondentes à matriz Jacobiana, são mantidas intactas. Através da simplificação da matriz Lagrangeana obtêm-se uma redução de memória utilizada, sem acarretar, com isso, uma perda sensível de eficiência na resolução do problema. Para viabilizar a análise, o problema de otimização foi modelado com as mesmas características do fluxo de carga convencional. A tabela 1 apresenta os resultados obtidos pelo FPO para o caso base sem contingência, e para o caso da contingência da LT entre as barras 1 e 2 do sistema IEEE-14. Conforme se observa na tabela 1 e na figura 6 , os valores encontrados por ambas as abordagens são praticamente os mesmos. O maior número de iterações é devido à simplificação da matriz Lagrangeana, a qual é compensada pela maior velocidade no processamento.

\section{MÉTODO PROPOSTO}

Neste trabalho também se propõe a determinação da margem de carregamento (MC) a partir do ponto de máximo carregamento de pré-contingência ( $\mathrm{PMC}_{\text {pré}}$ ) utilizando um FCC em que a tensão é utilizada como parâmetro. Entretanto, di-

\begin{tabular}{|c|c|c|c|}
\hline Contingência & Iterações & $\begin{array}{c}\lambda_{\max } \\
\text { (p.u.) }\end{array}$ & $\begin{array}{c}\text { Tensão crítica } \\
\text { (p.u.) /Nó }\end{array}$ \\
\hline $\begin{array}{c}\text { Nenhuma } \\
\text { (caso base) }\end{array}$ & 70 & 1.755 & $.613 / 14$ \\
\hline $\begin{array}{c}\text { LT entre as } \\
\text { barras 1 e 2 }\end{array}$ & 70 & 0.980 & $.840 / 14$ \\
\hline
\end{tabular}

Tabela 1: Resultados da simulação obtidas com o FPO para o sistema IEEE-14.

ferente do método proposto em Feng e Xu (2000), que utiliza o método de Newton-Raphson com fator de amortecimento para identificar e estimar a magnitude de tensão de pós-contingência da barra crítica, a qual será usada como parâmetro da continuação no $\mathrm{FCC}$, neste trabalho se propõe o uso de uma técnica de parametrização que possibilita a identificação da barra crítica de pós-contingência através da remoção gradual do ramo escolhido para contingência. Assim, o FCC parametrizado por tensão pode ser utilizado ao longo de todo o processo de obtenção da margem de carregamento. Nessa técnica, conforme apresentado na figura 7, para se evitar a divergência do FCC quando da aplicação da contingência, o ramo $k l$, correspondente à LT selecionada para avaliação da contingência, é parametrizado por um fator de escalonamento $(\mu)$, que é usado para a remoção gradual do ramo. Tanto a admitância série $\left(y_{k l}\right)$ quanto a susceptância $\left(y_{k l}^{s h}\right)$ da LT são gradualmente removidos através de sua multiplicação por $(1-\mu)$. Embora este procedimento não reflita necessariamente o comportamento transitório real do sistema após uma contingência, possibilita a determinação de um ponto de operação factível de pós-contingência.

$\mathrm{O}$ sistema de equações (1) do FC passa a ser representado por $G(\theta, \mathrm{V}, \lambda, \mu)=0$. Quando $\mu=0$ obtém-se o sistema original de equações do FC (1), e quando $\mu=1$ um novo sistema de equações é obtido, para o qual a o ramo $\mathrm{kl}$ foi completamente removido. A inclusão de $\mu$ como variável resultará em $n$ equações e $n+2$ incógnitas. Entretanto, prefixando o valor de $\mu$ ( $\mu=0$, caso base; $\mu=1$, contingência da LT), tem-se novamente $n$ equações e $n+1$ incógnitas. Assim, conforme descrito na seção 2, pode-se obter as respectivas curvas P-V por meio de um FCC (Ajjarapu e Christy, 1992; Chiang et alii, 1995; Alves et alii, 2002), ou seja, qualquer uma das $n+1$ incógnitas restantes pode ser definida como parâmetro. Seu valor $\left(\theta_{k}, V_{k}\right.$, ou $\left.\lambda\right)$ pode ser selecionado e, portanto, passase a ter novamente $n$ equações e $n$ incógnitas. É importante se observar nesse procedimento que, ao prefixar-se o valor de $\mu$ s estabelece-se a curva P-V a ser traçada, enquanto que com a prefixação de um dos demais parâmetro $\left(\theta_{k}, V_{k}\right.$, ou $\left.\lambda\right)$ traça-se a curva desejada. 


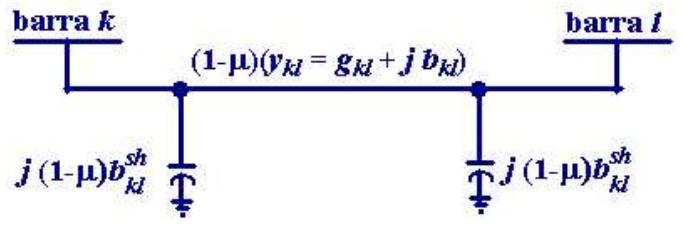

Figura 7: Definição da variável $\mu$ para realização da contingência da LT entre as barras $k$ e $l$.

\section{ANÁLISE DOS RESULTADOS}

Nos testes que se seguem a tolerância adotada para os mismatches foi de $10^{-4}$ p.u.. O primeiro ponto de cada curva foi obtido com inicialização flat start e considerando tap nominal. Os limites superior e inferior adotados para os tap foram 1,05 e 0,95 , respectivamente.

As cargas foram modeladas como potências constantes e o parâmetro $\lambda$ foi usado para simular o incremento de carga ativa e reativa, considerando fator de potência constante. $\mathrm{O}$ aumento de carga foi seguido por um aumento de geração usando $\lambda$. Com o objetivo de explicitar as características do método, considerou-se a influência dos limites de potência reativa $(Q)$ e de tap de transformadores. Os ajustes de tap nos transformadores OLTC são realizados com a inclusão da posição do tap como variável dependente, ao passo que as magnitudes de tensão das barras controladas foram consideradas como variáveis independentes (Peterson e Meyer, 1971). Os procedimentos para tratar os limites de $Q$ nas barras $P V$ e os limites dos tap, são os mesmos utilizados no método convencional de FC.

Observa-se que as análises foram efetuadas para os casos de LT e transformadores cujas remoções não resultassem em ilhamento ou remoção simultânea de geradores.

\subsection{Resultados Obtidos para o Sistema IEEE-14}

Conforme comentado anteriormente, o FCC parametrizado por $V_{14}$ divergirá quando a LT entre as barras 2 e 3 do sistema IEEE 14 (curva 3, figura 4) for completamente removida $(\mu=1)$, porque nesse caso não existirá solução local para as equações do FC. Assim, não será possível obter-se a MC para essa contingência a partir do $\mathrm{PMC}_{\text {pré}}$. De acordo com a figura 8 , se a remoção da LT for realizada prefixando gradualmente o valor de $\mu(0,5,0,8$, e 1,0$)$, e a tensão da barra $V_{14}$ for adotada como parâmetro, é possível obter-se os sucessivos pontos de máximo carregamento de pós-contingência $\left(\mathrm{PMC}_{\text {pós }}\right)$, e finalmente a MC pós-contingência, por meio de um procedimento similar àquele usado na seção 2 . Prefixando inicialmente o valor de $\mu$ em 0,5 e a tensão da barra
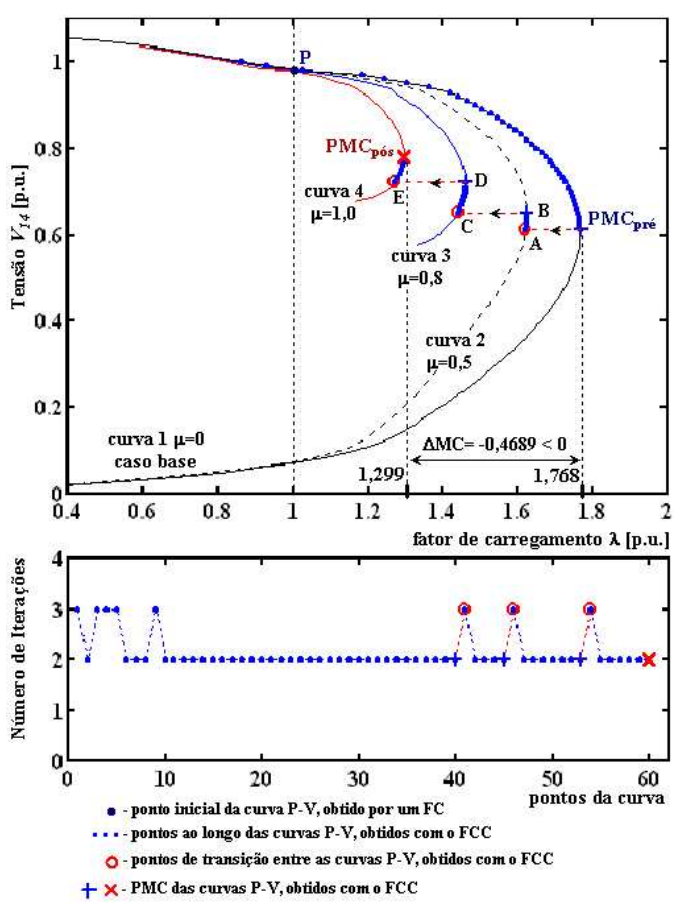

Figura 8: Contingência da LT entre as barras 2 e 3 do sistema IEEE-14: (a) Curvas P-V; e (b) número de iterações por ponto da curva.

$V_{14}$ no seu valor correspondente ao do $\mathrm{PMC}_{\text {pré }}(0,61$ p.u. $)$, calcula-se o ponto A na curva 2. A partir desse ponto o parâmetro $\left(V_{14}\right)$ é variado com passos de $+0,01$ p.u. e as respectivas soluções do FCC são obtidas, até que o ponto B seja alcançado. Esse procedimento é repetido para se obter os pontos C e D (para $\mu=0,8$ ), E (para $\mu=1,0)$ e o $\mathrm{PMC}_{\text {pós }}$, após o qual se calcula a MC de pós-contingência (que foi igual a 1,2991 p.u.). A redução na MC foi de $61,05 \%$. No caso dessa LT, como se pode ver na figura 8(b), foram necessárias ao todo 43 iterações, 9 iterações para obter os pontos $\mathrm{A}, \mathrm{C}$, e E e que correspondem às mudanças de curva em conseqüência da variação de $\mu$, e um total de 34 iterações gastos nas fases de refinamento.

\subsection{Resultados Obtidos para o Sistema IEEE-57}

A figura 9 apresenta as curvas P-V de duas barras (31 e 35), correspondentes à contingência da LT entre as barras $35 \mathrm{e}$ 36 do sistema IEEE 57, uma das mais severas contingências encontradas. Estas duas barras são apresentadas juntas na figura por serem as respectivas barras críticas de pré e pós- contingência, i.e., as que apresentam a maior taxa de variação de tensão próximo dos respectivos $\mathrm{PMC}$ de pré e pós-contingência, (Ajjarapu e Christy, 1992). A curva 1 cor- 


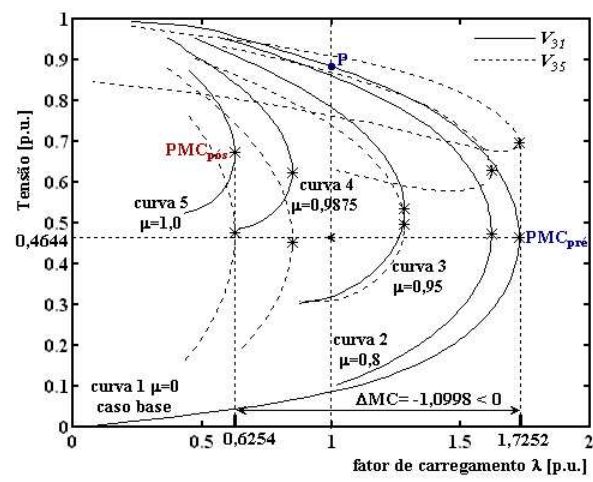

Figura 9: Curvas P-V para o caso base e para a contingência da LT entre as barras 35 e 36 do sistema IEEE-57.

responde ao caso base $(\mu=0)$ e a curva 5 à contingência completa ( $\mu=1)$ da LT. Primeiramente, adotou-se $\mu=0,8$ (curva 2), e incrementos graduais de 0,05 (curva 3) e 0,05/4 (curva 4). Da figura verifica-se que o FCC não encontrará um ponto de operação pós-contingência a partir do $\mathrm{PMC}_{\text {pré do caso base }}$ (curva 1), caso a tensão da barra 31 seja usada como parâmetro. Os argumentos são os mesmos que aqueles apresentados para a contingência da LT entre as barras 2 e 3 do sistema IEEE 14. Seguindo o mesmo procedimento já descrito para aquele caso, o método obtém êxito na determinação do $\mathrm{PMC}_{\text {pós }}$ e da MC de pós-contingência, conforme se pode verificar na figura 10(a). A redução na $\mathrm{MC}$ foi de $151,65 \%$. $\mathrm{Na}$ figura 10(b) pode-se ver que foram necessárias 56 iterações para se obter o $\mathrm{PMC}_{\text {pós }}$ a partir do $\mathrm{PMC}_{\text {pré}}$. Da figura verifica-se que o número de iterações necessário para passar de uma curva P-V para outra variou de 3 a 4 iterações (um total de 14 iterações), em geral, a maior parte das iterações (um total de 42 iterações) foram necessárias na fase de refinamento, i.e., no cálculo dos valores intermediários de

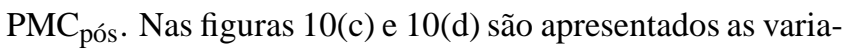
ções de alguns dos taps e as variações das potências reativas geradas em algumas barras $P V$, durante a aplicação do método proposto.

A figura 11 mostra, para a contingência da mesma LT, o desempenho da mudança do parâmetro utilizado pelo FCC proposto durante o procedimento de obtenção da MC de póscontingência. $\mathrm{O}$ critério adotado para troca de parâmetro foi considerar como parâmetro da continuação a tensão da barra que apresentasse a maior taxa de variação durante a passagem de uma curva P-V para outra. A identificação do novo parâmetro ocorreu durante a passagem da curva 2 para a 3 . $\mathrm{A}$ barra 35 foi a indicada e o valor de sua tensão no $\mathrm{PMC}_{\text {pós }}$ da curva 3 foi o valor prefixado. Observa-se na figura 11(a) que o ponto de operação obtido a seguir foi sobre a curva 5, próximo ao $\mathrm{PMC}_{\text {pós }}$ procurado. Com este procedimento de troca de parâmetro foram necessárias ao todo 31 iterações,
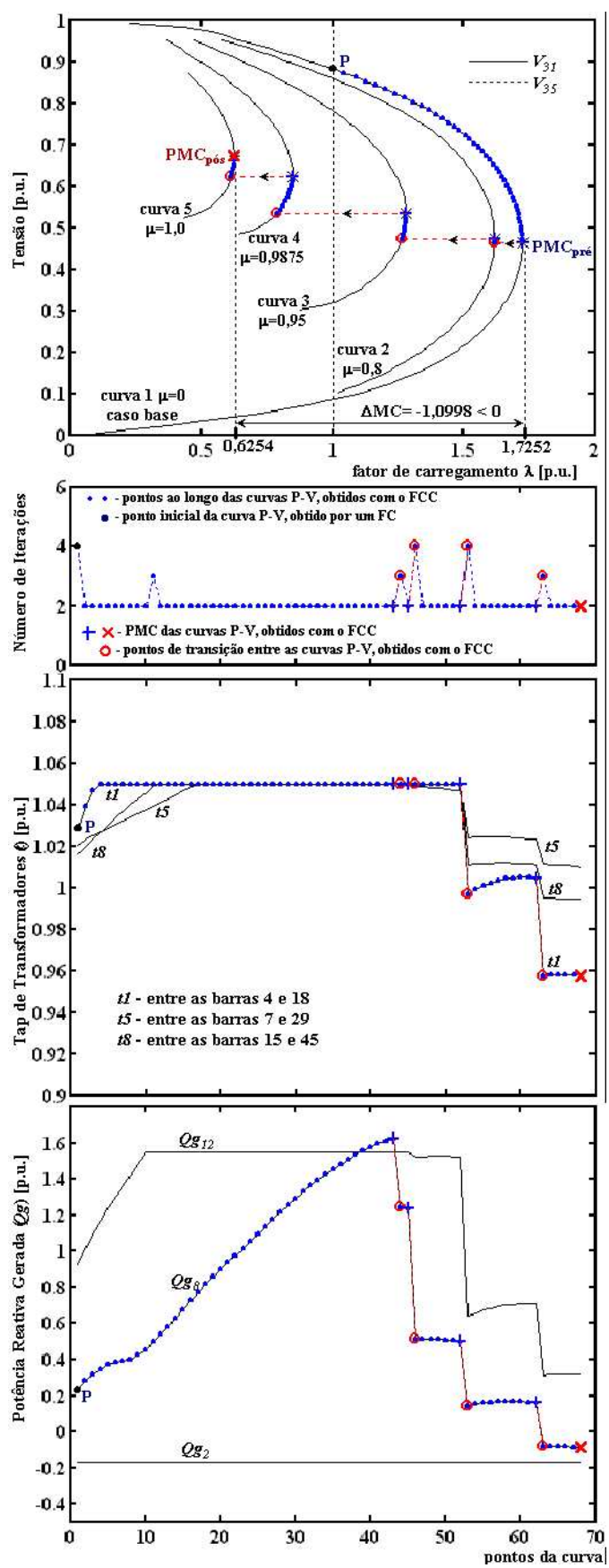

Figura 10: Contingência da LT entre as barras 35 e 36 do sistema IEEE-57: (a) Curvas P-V; (b) número de iterações por ponto da curva; (c) variação dos taps dos transformadores por ponto da curva; e (d) variação das potências reativas geradas nas barras $P V(2,8$, e 12$)$ por ponto da curva.

ou seja, resultou numa redução de 25 iterações. O número total de iterações necessárias para se passar de uma curva P-V para outra foi de 11 iterações (redução de 3 iterações), enquanto que o da fase de refinamento foi reduzido para 20. 

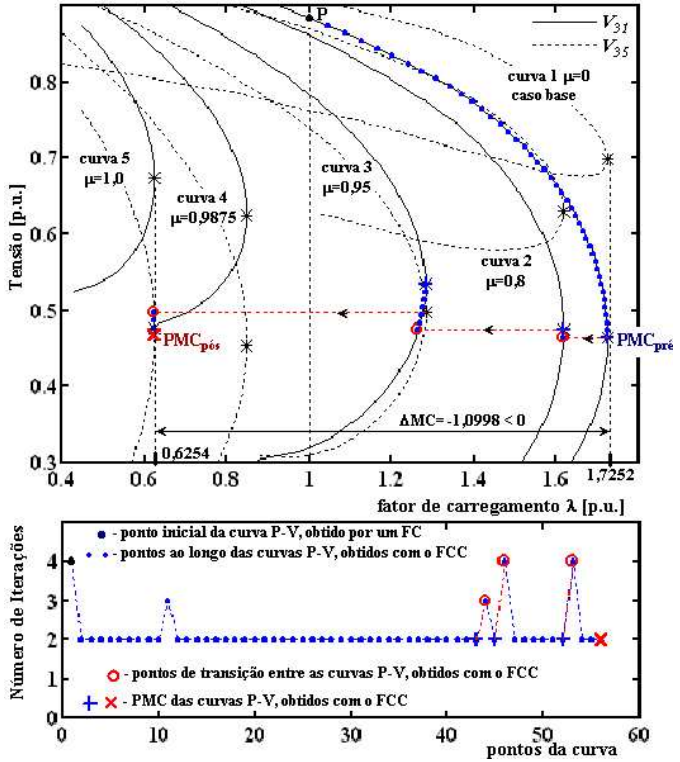

Figura 11: Desempenho da mudança do parâmetro utilizado pelo FCC proposto para obtenção da MC para a contingência da LT entre as barras 35 e 36 do sistema IEEE-57: (a) Curvas $\mathrm{P}-\mathrm{V}$; (b) número de iterações.

A figura 12 mostra, para a mesma LT da figura 11, o efeito do aumento do passo do parâmetro de tensão para 0,02 p.u. e da redução da tolerância dos mismatches de potência ativa e reativa para $10^{-3}$ p.u., utilizados pelo FCC proposto durante o procedimento de obtenção da MC de pós-contingência. Observa-se que o critério adotado para troca de parâmetro durante a passagem de uma curva $\mathrm{P}-\mathrm{V}$ para outra foi o mesmo usado para o traçado das curvas da figura 11. A identificação do novo parâmetro também ocorreu durante a passagem da curva 2 para a 3 , e a barra 35 foi novamente a indicada como o novo parâmetro. $\mathrm{O}$ valor de sua tensão no $\mathrm{PMC}_{\text {pós }}$ da curva 3 foi o valor prefixado. Observa-se na figura 12(a) que o ponto de operação obtido a seguir também foi sobre a curva 5 , próximo ao $\mathrm{PMC}_{\text {pós }}$ procurado, porém ligeiramente diferente (valor da magnitude da tensão um pouco maior que o da figura 11). Com a redução da tolerância dos mismatches de $10^{-4}$ para $10^{-3}$, e o aumento do passo do parâmetro de 0,01 p.u. para 0,02 p.u., o número total de iterações foi reduzido para 14, conforme apresentado na figura 12(b). Desse total foram necessárias 9 iterações para se passar de uma curva $\mathrm{P}-\mathrm{V}$ para outra e 5 durante as fases de refinamento. Portanto, resultou numa redução total adicional de 17 iterações, sem uma perda significativa da precisão do valor percentual de redução da MC, cujo valor obtido anteriormente era de $151,65 \%$, e agora é de $151,61 \%$.
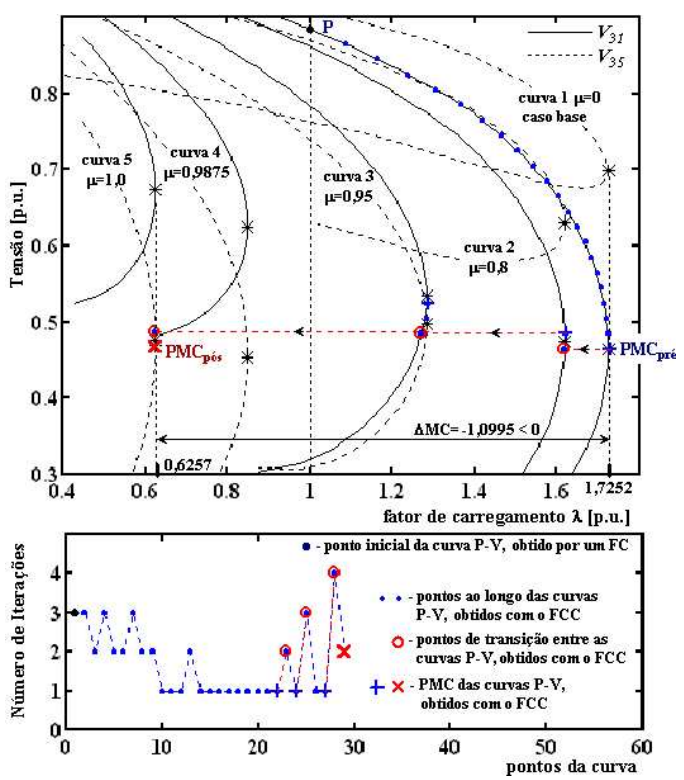

Figura 12: Desempenho da mudança do parâmetro utilizado pelo FCC proposto para obtenção da MC para a contingência da LT entre as barras 35 e 36 do sistema IEEE-57, redução da tolerância dos mismatches para $10^{-3}$ p.u. e aumento do passo do parâmetro $(V)$ para 0,02 p.u.: (a) Curvas P-V; (b) número de iterações.

\subsection{Resultados Obtidos para o Sistema IEEE-118}

Para o caso base este sistema não apresentou nenhuma contingência de LT ou transformador que resultasse numa MC negativa, i.e., cujo fator de carregamento $(\lambda)$ fosse inferior a 1 p.u.. Entretanto, são apresentadas as análises efetuadas para duas contingências, uma de transformador da LT 8 (localizado entre as barras 5 e 8, responsável pela menor MC encontrada) e outra da LT 116 (localizada entre as barras 69 e 75), em virtude das características peculiares apresentadas por esse sistema as quais caracterizam bem as dificuldades numéricas apresentadas durante a aplicação do método proposto.

As figuras 13 e 14 mostram, para a contingência do transformador da LT 8, o desempenho da mudança do parâmetro utilizado pelo FCC proposto durante o procedimento de obtenção da MC de pós-contingência. Foram consideradas as tensões de duas barras como parâmetro da continuação, a tensão do caso base de uma barra de carga qualquer, barra 9 , e a tensão do caso base da barra crítica, barra 13. O objetivo é mostrar que independente da tensão escolhida como parâmetro sempre será possível se obter o $\mathrm{PMC}_{\text {pós }}$. O critério adotado para troca de parâmetro foi o mesmo adotado para o IEEE-57, i.e., considerar como parâmetro da continuação a tensão da barra que apresentasse a maior taxa de variação 
durante a passagem de uma curva P-V para outra, sendo que o seu correspondente valor de tensão no PMC da respectiva curva deve ser o valor a ser mantido fixo durante a passagem de uma curva para a outra.

A figura 13 considera a tensão da barra $9\left(V_{9}\right)$ como parâmetro inicial. No detalhe da figura 13(b) pode-se ver a curva P-V da barra 9 para a contingência total $(\mu=1)$ do transformador da LT 8, onde verifica-se que não se pode apenas fixar sua tensão no correspondente valor do $\mathrm{PMC}_{\text {pré do caso base }}$
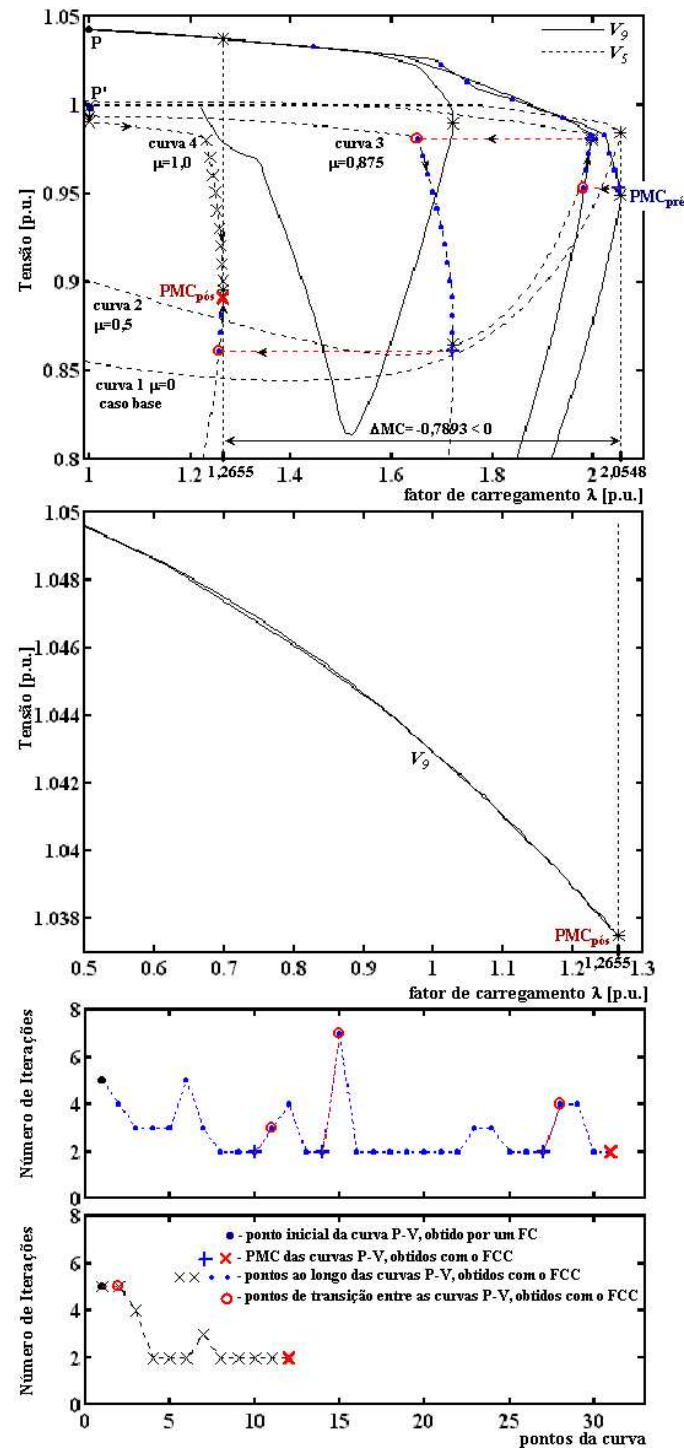

Figura 13: Desempenho da mudança do parâmetro utilizado pelo FCC proposto para obtenção da MC para a contingência do transformador da LT 8: (a) Curvas P-V; (b) detalhe da curva P-V; (c) número de iterações; (d) número de iterações, partindo do caso base $(\lambda=1)$ convergido e aplicando contingência da LT.
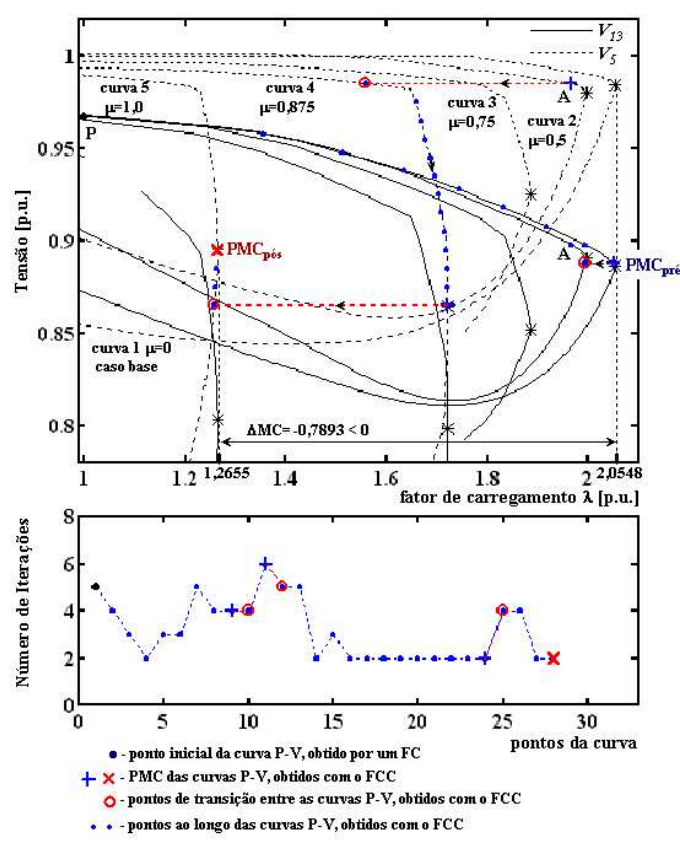

Figura 14: Desempenho da mudança do parâmetro utilizado pelo FCC proposto para obtenção da MC para a contingência do transformador da LT 8, inicializando com a tensão da barra crítica, barra 13, do caso base: (a) Curvas P-V; (b) número de iterações.

e considerar $\mu=1$, porque não existirá solução das equações do FCC nestas condições. Entretanto com o procedimento adotado mostra-se que é possível obter o $\mathrm{PMC}_{\text {pré}}$, mesmo escolhendo inicialmente a tensão desta barra como parâmetro. Assim, o valor inicial considerado para este parâmetro é o seu valor no $\mathrm{PMC}_{\text {pré }}$. Partindo-se do caso base $(\mu=0)$ adotou-se inicialmente um valor igual a 0,5 para $\mu$ (curva 2), os demais valores foram calculados considerando $\Delta \mu=0,5 \mathrm{e}$ $\mu=1-\Delta \mu / 4=0,875$ (curva 3), e finalmente a contingência total do elemento ( $\mu=1$, curva 4 ). A identificação do novo parâmetro ocorreu durante a passagem da curva 1 para a 2 . A barra 5 foi a indicada e o valor de sua tensão no $\mathrm{PMC}_{\text {pós }}$ da curva 2 foi obtido variando a tensão do parâmetro $\left(V_{9}\right)$ com passos de $+0,01$ p.u.. Então, o seu valor é prefixado para a obtenção do ponto sobre a curva 3 . Observe na figura 13(a) que o ponto de operação obtido sobre a curva 3 não se encontra próximo ao seu $\mathrm{PMC}_{\text {pós }}$. Assim, a partir desse ponto o parâmetro $\left(V_{5}\right)$ é variado com passos de $-0,01$ p.u. e as respectivas soluções do FCC são obtidas, até que o correspondente $\mathrm{PMC}_{\text {pós }}$ da curva 3 seja alcançado. Observa-se que durante a passagem da curva 2 para a curva 3 a barra 5 foi novamente a indicada como parâmetro. Após se obter o PMC da curva 3, a tensão da barra 5 é fixada no seu valor correspondente, e o valor de $\mu$ é fixado em 1 (contingência total). Com este procedimento de troca de parâmetro foram necessárias ao todo 56 iterações. Um total de 14 iterações foi necessário para pas- 
sar de uma curva P-V para outra, enquanto que as fases de refinamento exigiram 42 iterações. A MC para esta contingência é de $26,55 \%$. Na figura 13 (d) pode se ver que nesse caso o número total de iterações necessárias para se obter o $\mathrm{PMC}_{\text {pós }}$, partindo do caso base $(\lambda=1)$ convergido e aplicando contingência da LT, foi igual a 28. Portanto, maior que o número total de iterações necessárias para se determinar o PMC a partir do $\mathrm{PMC}_{\mathrm{pré}}$.

A figura 14 considera a tensão da barra crítica $\left(V_{13}\right)$, barra 13 do caso base, como parâmetro inicial. Seguindo o mesmo procedimento, a barra 5 é novamente a indicada como parâmetro para a obtenção do ponto sobre a próxima curva, no caso curva 4. Neste caso foram necessárias ao todo 55 iterações. O número total de iterações necessárias para passar de uma curva $\mathrm{P}-\mathrm{V}$ para outra foi igual a 13 , enquanto que as fases de refinamento exigiram 42 iterações. Portanto, obteve-se praticamente o mesmo desempenho.

A figura 15 considera novamente a tensão da barra crítica $\left(V_{13}\right)$, barra 13 do caso base, como parâmetro inicial. O critério para troca de parâmetro foi mantido, ou seja, considerouse como parâmetro da continuação a tensão da barra que apresentasse a maior taxa de variação durante a passagem de uma curva P-V para outra. Entretanto, objetivando reduzir o número de iterações e evitar a determinação dos PMC intermediários, adotou-se que o valor da tensão da barra crítica do caso base será o valor adotado para todas as barras indicadas até que $\mu$ seja igual a 1, ou seja, o PMC será determinado somente para a curva correspondente à contingência total. Com esse procedimento o objetivo de reduzir o número total de iterações foi alcançado, como se pode verificar na figura $15(\mathrm{c})$, de onde se constata que o número total de iterações foi 31 , sendo 20 iterações necessárias para passar de uma curva P-V para outra. Entretanto, deve-se ressaltar que foi necessária uma pequena mudança de estratégia para se obter o ponto $\mathrm{D}$, isto porque o método divergiu ao tentarse obter diretamente o ponto $\mathrm{D}$ na curva 4 a partir do ponto A na curva 2. Primeiro foi necessário uma redução no incremento de $\mu$, i.e., foi necessário um outro ponto intermediário, situado na curva de $\mu=0,75$. Também, conforme pode-se ver no detalhe da figura 15(b), ao obter-se o ponto A, a tensão da barra 5 (ponto A' na curva 2), indicada como o novo parâmetro, apresentava o valor de 0,9767 p.u. (oito vezes o valor do passo de tensão 0,01 p.u. adotado na fase de refinamento para a obtenção do PMC de uma curva P-V), o que exigiu um passo de ajuste intermediário com 7 iterações para alcançar o ponto B na mesma curva. A partir do ponto B pode-se obter o ponto $\mathrm{C}$ sobre a curva 3 e a seguir o ponto $\mathrm{D}$, mantendo-se o valor do parâmetro $V_{5}$ fixo em 0,8880 p.u.. Observa-se que a fase de refinamento só foi considerada na curva correspondente a $\mu=1$. Por outro lado, conforme se mostra na figura 16, o uso da tensão da barra 9 mostrou-se mais eficiente, no tocante a passagem de uma curva para ou- tra, porque após a obtenção do ponto sobre a curva 2, a tensão da barra 5 encontrava-se mais próxima da tensão da barra 9. Assim, o processo pôde prosseguir sem a necessidade de passos de ajuste intermediário até obter o $\mathrm{PMC}_{\text {pós }}$. Foram realizadas ao todo 29 iterações, sendo 17 iterações necessárias para passar de uma curva $\mathrm{P}-\mathrm{V}$ para outra. Assim, no geral, pode-se considerar que os dois parâmetros apresentaram praticamente o mesmo desempenho, i.e., a necessidade de um passo de ajuste intermediário não implica num aumento sensível no número total de iterações. Observa-se também, que com este procedimento obtém-se praticamente o mesmo desempenho que o alcançado partindo do caso base $(\lambda=1)$ convergido e aplicando contingência da LT e que foi igual a 28, conforme apresentado na figura 13(d). Portanto, seria possível, a princípio, se reduzir o número total de iterações evitando-se a determinação dos PMC intermediários. Entretanto, como será visto a seguir, esse procedimento nem sempre é possível, e assim foi descartado em função da garantia de sucesso na obtenção do $\mathrm{PMC}_{\text {pós }}$.

As figuras 17 e 18 apresentam, para a contingência da LT 116, o desempenho da mudança do parâmetro utilizado pelo FCC proposto durante o procedimento de obtenção da MC de pós-contingência. Novamente foram consideradas as tensões do caso base das duas barras, barra 9 e barra 13 (a barra crítica) como parâmetro da continuação. É importante notar que no caso desta contingência em particular, bem como para muitas outras, as respectivas curvas $\mathrm{P}-\mathrm{V}$, para o caso base $(\mu=0)$ e para contingência de $50 \%(\mu=0,5)$, de todas as barras, são praticamente coincidentes, conforme pode-se verificar no detalhe da figura 17(a). Também, como pode-se verificar dessas duas figuras, tanto a tensão da barra 9 quanto da barra 13 apresentam, para $\mu>0,5$, um comportamento similar ao apresentado no detalhe da figura 13(b), ou seja, não se pode apenas fixar qualquer uma destas tensões no corres-

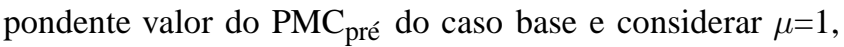
porque não existirá solução das equações do FC nestas condições. Assim, apesar da MC do sistema após esta contingência ser de $91,79 \%$, o uso da tensão da barra 9 como parâmetro e a fixação do valor do $\mu$ em 1 impossibilitaria a classificação quanto ao grau de severidade desta contingência. Entretanto, com o critério adotado para troca de parâmetro, ou seja, o de considerar como parâmetro da continuação a tensão da barra que apresentasse a maior taxa de variação durante a passagem de uma curva $\mathrm{P}-\mathrm{V}$ para outra, sendo que o seu correspondente valor de tensão no PMC deve ser o valor a ser mantido fixo durante a passagem de uma curva para a outra, possibilita a obtenção do $\mathrm{PMC}_{\text {pós }}$ sem nenhuma dificuldade numérica. O número total de iterações necessárias, 51 e 61, podem ser vistos nas figuras 17(b) e 18(b), respectivamente. Desse total, foram necessárias, em ambos os casos, 13 iterações para passar de uma curva P-V para outra, enquanto que o correspondente as fases de refinamento foram 38 e 48 iterações, respectivamente. Observa-se que o uso da tensão da 

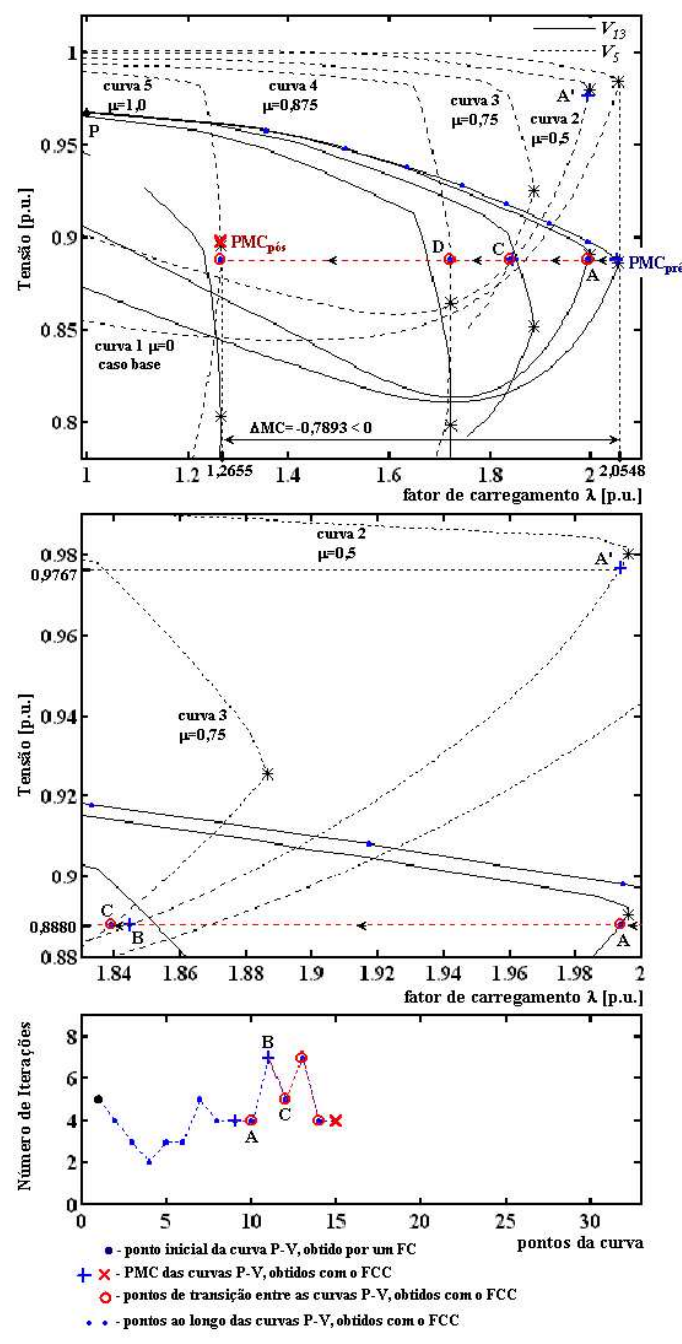

Figura 15: Desempenho da mudança do parâmetro utilizado pelo FCC proposto para obtenção da MC para a contingência do transformador da LT 8, inicializando com a tensão da barra crítica, barra 13, do caso base: (a) Curvas P-V; (b) número de iterações.

barra 9 como parâmetro resultou num menor número de iterações, ou seja, nem sempre o uso da tensão da barra crítica levará a um melhor desempenho. Na figura 17(d) pode se ver que nesse caso, e que corresponde à grande maioria das contingências, o número total de iterações necessárias para se obter o $\mathrm{PMC}_{\text {pós }}$, partindo do caso base $(\lambda=1)$ convergido e aplicando contingência da LT, foi igual a 90 . Portanto, maior que o número total de iterações necessárias para se determinar o $\mathrm{PMC}$ a partir do $\mathrm{PMC}_{\text {pré }}$.

Nas figuras 17(c) e 18(c) pode-se verificar que com a redução da tolerância dos mismatches de $10^{-4}$ para $10^{-2}$ p.u., e o aumento do passo do parâmetro de 0,01 p.u. para 0,02 p.u., o número total de iterações foi reduzido para 27 , e 38 , respecti-
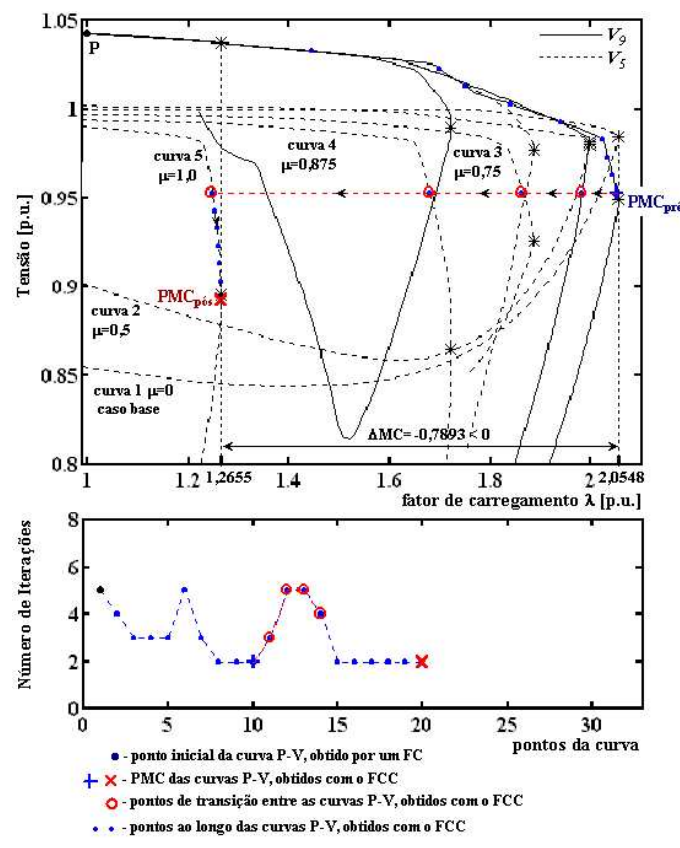

Figura 16: Desempenho da mudança do parâmetro utilizado pelo FCC proposto para obtenção da MC para a contingência do transformador da LT 8, inicializando com a tensão da barra 9 do caso base: (a) Curvas P-V; (b) número de iterações.

vamente. Desse total foram necessárias respectivamente, 11 e 9 iterações para se passar de uma curva P-V para outra, e 16 e 29 durante as fases de refinamento. Portanto, alcançouse uma redução total adicional de 24 e 23 iterações, sem uma perda significativa da precisão do valor percentual de redução da MC, cujo valor obtido anteriormente era de 91,79\%, e agora é de $92,13 \%$.

\section{CONCLUSÕES}

Neste trabalho apresentou-se um método para a análise estática de contingências em sistemas elétricos de potência. $\mathrm{O}$ método possibilita a determinação da margem de estabilidade após a contingência de qualquer transformador ou linha de transmissão. A convergência do FCC é assegurada através do uso de uma técnica de parametrização em que os parâmetros do modelo equivalente $\pi$, do transformador ou da linha de transmissão selecionada para avaliação da contingência, são parametrizados por um fator de escalonamento que possibilita sua remoção gradual. O procedimento usado para a troca de parâmetro durante a passagem de uma curva $\mathrm{P}-\mathrm{V}$ para a outra é similar àqueles encontrados na literatura. Uma vez que requer poucas modificações dos métodos de FCC já existentes, o método apresentado facilita a sua implementação computacional. 

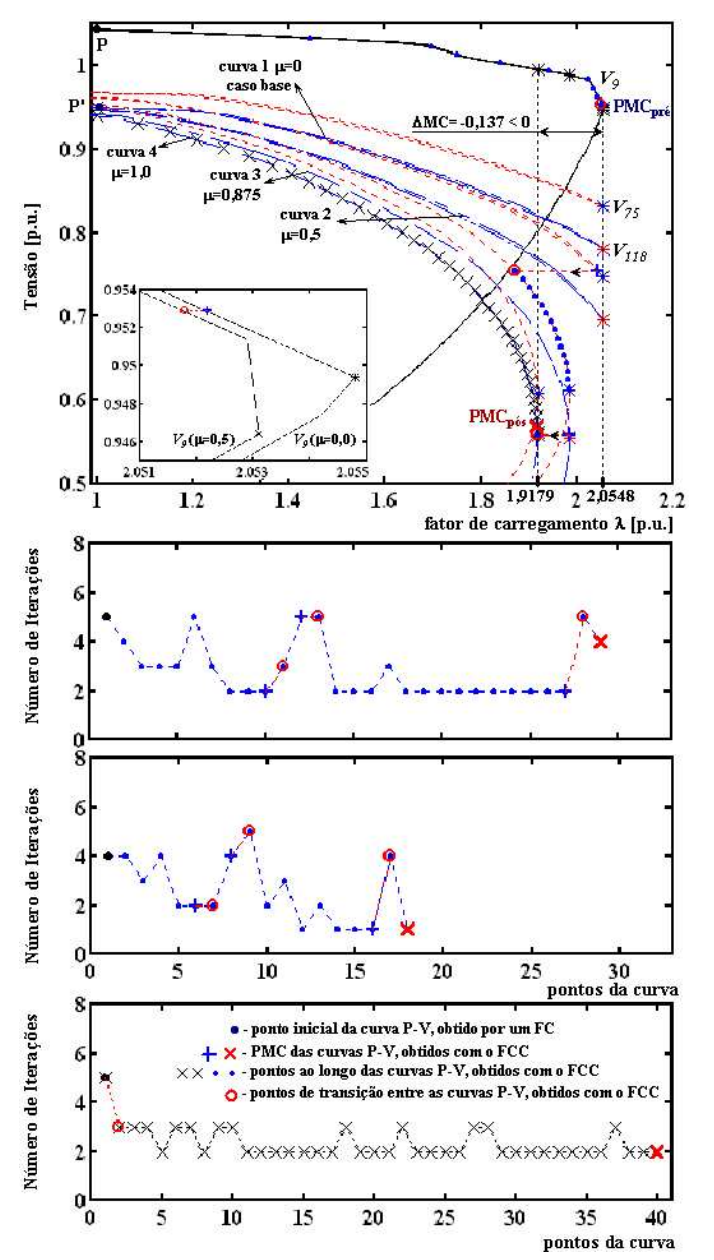

Figura 17: Desempenho da mudança do parâmetro utilizado pelo FCC proposto para obtenção da MC para a contingência da LT 116 entre as barras 69 e 75, inicializando com a tensão da barra 9 do caso base: (a) Curvas P-V; (b) número de iterações; (c) número de iterações com tolerância de $10^{-2}$ p.u. para os mismatches e passo de 0,02 p.u. para o parâmetro $(V)$; (d) número de iterações, partindo do caso base $(\lambda=1)$ convergido e aplicando contingência da LT.

\section{AGRADECIMENTOS}

Os autores agradecem ao CNPq, CAPES e FAPESP pelo suporte financeiro a este trabalho.

\section{REFERÊNCIAS}

Ajjarapu, V. e Christy, C. (1992). The Continuation Power Flow: A Tool for Steady State Voltage Stability Analysis, IEEE Trans. on Power Systems, vol. 7, pp. 416-423.

Alves, D. A., da Silva, L. C. P., Castro, C. A. e da Costa, V. F. (2002). Esquemas Alternativos para o Passo de
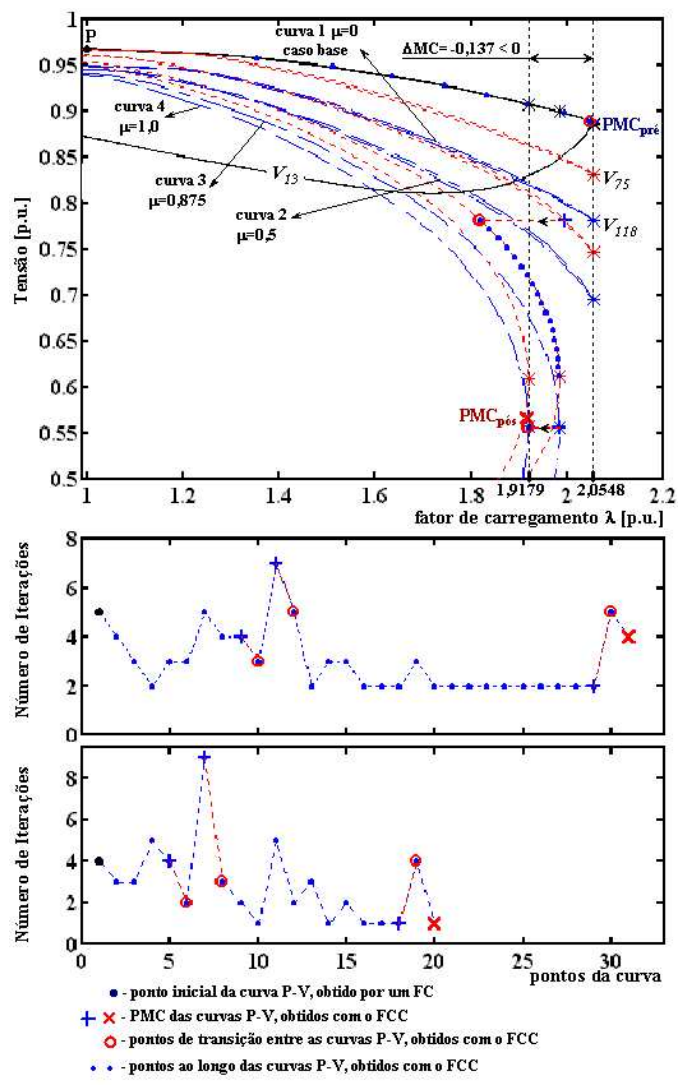

Figura 18: Desempenho da mudança do parâmetro utilizado pelo FCC proposto para obtenção da MC para a contingência da LT 116 entre as barras 69 e 75, inicializando com a tensão da barra crítica (barra 13) do caso base: (a) Curvas P-V; (b) número de iterações; (c) número de iterações com tolerância de $10^{-2}$ p.u. para os mismatches e passo de 0,02 p.u. para o parâmetro $(V)$.

Parametrização do Método da Continuação Baseados em Parâmetros Físicos, Revista Controle \& Automação, vol. 13, no. 3, pp 275-289.

Alves, D. A., da Silva, L. C. P., Castro, C. A. e da Costa, V. F. (2004). Parameterized Fast Decoupled Power Flow Methods for Obtaining the Maximum Loading Point of Power Systems - Part-I: Mathematical Modeling, Electric Power Systems Research, vol 69, no. 1, pp 93-104.

Barbosa, L. V. e Salgado R. (2001). Unsolvable Power Flow Analysis - An Approach Based on Interior Point NonLinear Optimization Methods, IEEE Power Tech Conference, Porto, Portugal.

Cañizares, C. A. (1995), Conditions for Saddle-Node Bifurcations in AC/DC Power Systems, Electric Power Energy Systems, vol. 17, pp. 61-68. 
Castro, C. A. e L. M. C. Braz (1997). Uma Nova Abordagem para a Solução do Problema de Fluxo de Carga pelo Método de Newton com Otimização de Passo, Revista Controle \& Automação, vol. 8, no. 3, pp. 121-133.

Chiang, H. D., Li, H., Yoshida, H., Fukuyama, Y. e Nakanishi, Y. (1999). The Generation of ZIP-V Curves for Tracing Power System Steady State Stationary Behavior due to Load and Generation Variations, IEEE PES Summer Meeting, Edmonton, Canada, pp. 647-651.

Chiang, H. D., Flueck, A. J., Shah, K. S. e Balu, N. (1995). CPFLOW: A Practical Tool for Tracing Power Systems Steady-State Stationary Behavior due to Load and Generation Variations, IEEE Trans. Power Systems, vol. 10, pp. 623-633.

Chiang, H. D., Wang, C. S. e Flueck, A. J. (1997). LookAhead Voltage and Load Margin Contingency Selection Functions for Large Scale Power Systems, IEEE Trans. Power Systems, vol. 12, pp. 173-180.

Da Costa, G. R. M., Langona, M K., Alves, D. A. (1998). A New Approach to the Solution of the Optimal Power Flow Problem Based on the Modified Newton's Method Associated to an Augmented Lagrangian Function, Proceedings of the International Conference on Power System Technology, Beijing, China, pp. 909-913.

Ejebe, G. C., Irissari, G. D., Mokhatari, S., Obadina, O., Ristanovic, P. e Tong, J. (1996). Methods for Contingency Screening and Ranking for Voltage Stability Analysis of Power Systems, IEEE Trans. on Power Systems, vol. 11, pp. 350-356.

Feng, Z. e Xu, W. (2000). Fast Computation of PostContingency System Margin for Voltage Stability Assessments of Large-Scale Power Systems, IEE Proceedings-Generation, Transmission and Distribution, vol. 147, no 2, pp. 76-80.

Flatab $\emptyset$, N., Ognedal, R., e Carlsen, T. (1990). Voltage Stability Condition in a Power Transmission System Calculated by Sensitivity Methods, IEEE Trans. on Power Systems, vol. 5, no. 4, pp. 1286-1293.

Freris L. L. e Sasson A. M. (1968). Investigation of the LoadFlow Problem, Proceeding of IEE, vol. 115, no. 10, 1459-1470.

FTCT (1999). Critérios e Metodologias Estabelecidos no Âmbito da Força-Tarefa "Colapso de Tensão" do GTAD/SCEL/ GCOI para Estudos de Estabilidade de Tensão nos Sistemas Interligados Norte/Nordeste, Sul/Sudeste e Norte/Sul Brasileiros, XV SNPTEE, Foz do Iguaçu, PR, Brasil.
Granville, S., Mello, J. C. O., Melo, A. C. G. (1996). Application of Interior Point Methods to Power Flow Unsolvability. IEEE Trans. Power Systems, vol. 11, no. 2, pp. 1096-1103.

Granville, S., Latorre, M. L., Ojeda, J. R. (1996-2). Colapso de Tensão e Fluxo de Potência Ótimo em Sistema de Potência, Eletroevolução, pp. 47-53.

Greene, S., Dobson, I. e Alvarado, F. L. (1999). Contingency Ranking for Voltage Collapse via Sensitivities from a Single Nose Curve, IEEE Trans. on Power Systems, vol. 14, pp. 232-240.

IEEE Power System Stability Committee (IEEE-SSC), Special Publication (1999). Final Draft: Voltage Stability Assessment, Procedures and Guides, http://www.power.uwaterloo.ca.

ONS (2001). ONS Network Procedures-Guidelines and Criteria to Electrical Studies.

Overbye, T. J. (1994). A Power Flow Measure for Unsolvable Cases, IEEE Trans. on Power Systems, vol. 9, no. 3, pp. 1359-1365.

Peterson N. M. e W. S. Meyer (1971). Automatic Adjustment of Transformer and Phase-Shifter Taps in the Newton Power Flow, IEEE Trans. on PAS, vol. PAS-90, no. 1, pp. 103-108.

Rider, M. J., Castro, C. A., Paucar, V. L., Garcia, A.V. (2004). Higher-Order Interior Point Method for Minimising Load-Shedding in a Competitive Electric Power Market. IEE Prooc. Generation Transm. Distrib., vol. 151, no. 4, pp. 433-440.

Taylor, C. W. (1994). Power System Voltage Stability, A MacGraw-Hill, Inc., New York, EUA.

Torres, G. L., Quintana, V. H., Carvalho, M. A. (2002). Higher-Order Interior Point Methods for Computing Minimum Load Shedding and Maximum Loadability of Power Systems. VIII Simpósio de Especialistas em Planejamento da Operação e Expansão Elétrica (SEPOPE), Brasília (DF), Brasil, IP-057.

Van Cutsem, T. e Mailhot, R. (1997). Validation of a Fast Voltage Stability Analysis Method on the HydroQuébec System, IEEE Trans. on Power Systems, vol. 12, pp. 282-292.

Web site da Universidade de Washington, The Standard IEEE 118 Test Data, http://www.ee.washington.edu/research/pstca/, disponível em 2003. 
WSCC-Reactive Power Reserve Work Group (RRWG) (1998). Final Report: Voltage Stability Criteria, Undervoltage Load Shedding Strategy, and Reactive Power Reserve Monitoring Methodology, 154p.

Yokoyama, A. e Sekine, Y. (1989). A Static Voltage Stability Index Based on Multiple Load Flow Solutions, Proceedings of Bulk Power System Voltage PhenomenaVoltage Stability and Security Conference, Missoruri, EUA. 